\title{
Faire l'inventaire du patrimoine ferroviaire : expériences et méthodes
}

Paul Smith

\section{(2) OpenEdition \\ Journals}

Édition électronique

URL : https://journals.openedition.org/rhcf/705

DOI : 10.4000/rhcf.705

\section{Éditeur}

Rails \& histoire

Édition imprimée

Date de publication : 1 novembre 2009

Pagination : 10-13

ISSN : 0996-9403

Référence électronique

Paul Smith, «Faire l'inventaire du patrimoine ferroviaire : expériences et méthodes », Revue d'histoire des chemins de fer [En ligne], 40 | 2009, mis en ligne le 01 novembre 2011, consulté le 22 avril 2022 URL : http://journals.openedition.org/rhcf/705 ; DOI : https://doi.org/10.4000/rhcf.705 
Paul SMITH

\section{Faire l'inventaire du patrimoine ferroviaire : expériences et méthodes}

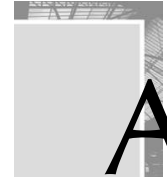

ccueillie, passage Colbert, dans les locaux parisiens de l'Institut national du Patrimoine, la journée d'études du 22 octobre 2008 fut organisée conjointement par l'Association pour l'histoire des chemins de fer en France et la mission Inventaire général du patrimoine culturel, qui assure les compétences de l'État dans ce domaine au sein de la sous-direction de l'archéologie, de l'ethnologie, de l'inventaire et du système d'information (direction de l'architecture et du patrimoine, ministère de la culture et de la communication). L'ambition de cette journée d'études était de provoquer des échanges et d'établir des liens entre deux communautés scientifiques, celle, d'une part, des historiens et des amis des chemins de fer en France réunis dans l'AHICF et celle des chercheurs chargés, dans les régions, de l'Inventaire général du patrimoine culturel, de l'autre.

Il ne s'agissait pas, bien évidemment, de la première rencontre entre l'univers ferroviaire et les services chargés de la culture. Sans remonter à 1926 et à la première protection d'un bâtiment ferroviaire au titre de la législation 
sur les monuments historiques ${ }^{1}$, on se souvient, peut-être, au début des années 1980, des conventions "Transports-Culture " établies entre la SNCF et la direction du patrimoine, aboutissant, entre autres résultats moins durables, à toute une série de mesures de protection juridique du patrimoine immobilier du chemin de fer : des gares, surtout, mais aussi des viaducs, des têtes de tunnel, des rotondes, des ateliers d'entretien, des cités cheminotes... Aujourd'hui, à côté de 524 spécimens du matériel roulant protégés, on peut compter 115 " monuments " ferroviaires classés ou inscrits, des bâtiments et sites encore en activité ou désaffectés et placés, dans leur département respectif, sous la surveillance d'agents du ministère de la culture ${ }^{2}$.

Mais si la notion de monument historique est assez familière, celle d'Inventaire général l'est peut-être moins : pour la plupart des participants à la journée d'études du 22 octobre, ainsi que pour les lecteurs de ces pages de la Revue d'histoire des chemins de fer, cette communauté scientifique est sans doute moins connue. L'Inventaire général du patrimoine culturel est le nom actuel de l'entreprise documentaire, et du service qui en a la charge, créés par André Malraux en 1964, sous le titre d'Inventaire général des monuments et des richesses artistiques de la France. Sa mission : recenser, étudier et faire connaître le patrimoine de la nation, ou, plus précisément aujourd'hui, aux termes de la loi du 13 août 2004, « les éléments du patrimoine qui présentent un intérêt culturel, historique ou scientifique ". Pour s'acquitter de la partie " faire connaitre " de cette mission, l'Inventaire a recours à des moyens classiques, comme des expositions et des publications, mais nourrit surtout en notices informatisées et en dossiers en ligne les bases de données nationales sur le patrimoine qui sont Mérimée, pour le patrimoine architectural, et Palissy pour le patrimoine des objets mobiliers ${ }^{3}$.

\footnotetext{
1- Il s'agit de l'entrée du tunnel ferroviaire du Fréjus, alors désaffectée, en Savoie. La dernière protection en date, l'inscription de la gare des houillères à Fresne-sur-Escaut dans le Nord, est intervenue dans le cadre de la candidature du bassin minier du Nord Pas-de-Calais pour une inscription sur la liste du patrimoine mondial. Sur l'histoire de la protection au titre des monuments historiques des bâtiments et infrastructures liés au chemin de fer, voir Paul Smith, «Le patrimoine ferroviaire en France : soixante-dix ans de protection juridique », in « Le patrimoine ferroviaire : enjeux, bilans et perspectives ", Actes du 6 colloque de l'AHICF (Mulhouse, 23-26 septembre 1998), en collaboration avec le CILAC, Comité d'information et de liaison pour l'archéologie, l'étude et la mise en valeur du patrimoine industriel, et le Musée français du Chemin de fer, Revue d'bistoire des chemins de fer, n 20-21, 1999, p. 329-347.

2- La liste des monuments historiques des chemins de fer est tenue à jour sur le site de l'AHICF, http://www.ahicf.com/patrimoine.htm.

3- http://www.culture.gouv.fr/culture/inventai/patrimoine/
} 
Depuis bientôt cinquante ans, donc, ce service, fort aujourd'hui d'environ trois cents agents à Paris et dans les régions, accomplit sa triple mission en accompagnant l'évolution des perceptions du patrimoine - on parle souvent de son " explosion » - et en contribuant à cette évolution, la devançant même, notamment dans l'attention accordée depuis le début des années 1980 à ce "nouveau " patrimoine qui est celui des bâtiments, des sites et des machines légués par l'industrie. L'Inventaire a également accompagné l'évolution administrative du pays, suivant, dans la délimitation des territoires sur lesquels il vient à appliquer ses méthodes de recensement et de documentation, l'émergence de l'intercommunalité. Depuis la loi de décentralisation de 2004, les services régionaux de l'inventaire général, jusqu'alors rattachés aux directions régionales des affaires culturelles, sont passés sous la tutelle des conseils régionaux, nouvelle donne qui, on le verra, donne aux inventaires du patrimoine ferroviaire une nouvelle actualité.

Pratiquement depuis ses origines, toutefois, dans ses différents types d'enquête, topographiques ou thématiques, l'Inventaire général s'est attaché à étudier et à documenter des éléments du patrimoine ferroviaire. Dans la base Mérimée, la notice la plus ancienne sur ce patrimoine date d'une enquête menée sur le terrain en 1969. Elle concerne la gare de Bolbec dans le département de la Seine-Maritime, qui n'existait déjà plus au moment de l'enquête! Depuis lors, et suivant l'étude entreprise par Guillaume Simon en préparation de la journée d'études du 22 octobre 4 , on peut dénombrer 568 notices Mérimée consacrées à des éléments bâtis du chemin de fer, très majoritairement des gares : pas loin de 400 notices, chiffre qu'on peut mettre en face de celui des 2200 bâtiments voyageurs actuellement exploités par la SNCF - sans parler des milliers de gares ayant existé jusque dans les plus petites communes - et qu'on pourrait aussi rapporter au nombre de communes touchées aujourd'hui par une enquête de l'Inventaire (environ une sur trois). Pour la plupart, ces gares sont vues, pour ainsi dire, du côté de la ville et non pas du côté des voies, l'Inventaire avançant par aire d'étude (en général, au début du moins, le canton) et rencontrant ces bâtiments comme autant d'œuvres individuelles au sein de ce périmètre. Depuis les années 1990, cependant, on voit venir un nombre assez significatif de notices indexées sous la dénomination de "voie ferrée ", rendant compte d'études qui cherchent à appréhender le patrimoine du chemin de fer non pas en termes d'une accumulation d'édifices isolés -

4- Cette étude, publiée dans ces actes, est également consultable sur le site de la revue en ligne, In-Situ, Revue des Patrimoines, $\mathrm{n}^{\circ}$ 10. (http://www.revue.inventaire.culture.gouv.fr/insitu/insitu/ article.xsp?numero=10\&id_article=simon-984) 
une gare qui n'en cache pas une autre - mais en termes de réseau, avec des éléments reliés entre eux, de manière physique et organisationnelle, le long d'une voie ou d'un tronçon de voies.

C'est également par voies et toujours pour « faire connaître » que l'Inventaire a publié, dans ses différentes collections, trois ouvrages spécifiquement consacrés au patrimoine du chemin de fer : un «itinéraire » du patrimoine sur le Train Jaune en Languedoc-Roussillon, un " parcours » du patrimoine sur la ligne dite des Hirondelles entre Andelot et La Cluse en Franche-Comté et une « image " du patrimoine sur la première grande ligne en France de Paris à Rouen et au Havre. Enfin, dans la collection des « cahiers » du patrimoine, il faut mentionner aussi la publication récente sur la ville de Juvisy, agglomération en grande partie structurée par les réseaux de transport qui le traverse et où la gare représente un quart du territoire de la commune ${ }^{6}$.

Notices et dossiers électroniques, publications diverses, ces différents travaux font tous ressortir, parfois en négatif, les multiples difficultés que rencontrent les services de l'Inventaire - ou d'autres acteurs - dans l'étude du patrimoine ferroviaire. Quelles sources mobiliser ? Comment organiser les dossiers et les relier entre eux pour rendre compte de l'unité d'un réseau dans le temps et dans l'espace ? Comment traiter un patrimoine linéaire s'étendant sur plusieurs aires d'étude habituelles, voire sur plusieurs régions ? Comment documenter, et jusqu'à quel niveau de détail, une architecture en série issue de plans type ? Comment saisir les éléments d'un patrimoine souvent modeste - maisons de garde-barrière, postes d'aiguillage, signalétique, garde-corps, poteaux kilométriques...- mais qui donnent aux paysages du rail leur caractère particulier ? Comment, au-delà des emprises immédiates, mesurer et rendre perceptible - et de quel point de vue ? - l'impact historique, social et paysager du chemin de fer sur les territoires qu'il traverse : les quartiers de la gare avec leurs hôtels et cafés, les dépôts, les gares marchandises, les triages et ateliers

5- L'on consultera, par exemple, le dossier établi par Maryannick Chalabi et Chantal ArcherGaléa sur la voie ferrée du deuxième arrondissement de Lyon (référence Mérimée IA69000240). 6- Michel Wienin, Le Train Jaune, un chemin de fer d'exception, Inventaire général LanguedocRoussillon, 2000 (Itinéraires du patrimoine, $\mathrm{n}^{\circ}$ 226) ; Laurent Poupard, La Ligne des Hirondelles, un chemin de fer entre ciel et terre, d'Andelot à la Cluse, Jura-Ain, Inventaire général Franche-Comté, 2008 (Parcours du patrimoine, $n^{\circ}$ 336) ; Hélène Bocard et al., De Paris à la Mer, la ligne de chemin de fer Paris-Rouen-Le Havre, Inventaire général Île-de-France et Haute-Normandie, 2005 (Images du patrimoine $\left.\mathrm{n}^{\circ} 239\right)$; Antoine Le Bas, Jwisy-sur-Orge. Un territoire, des réseaux, Inventaire général Île-de-France, 2007 (Cahiers du patrimoine n ${ }^{\circ} 8$ ). 
d'entretien, les cités de cheminots, les sites industriels embranchés et induits, le relief même, sculpté par des ouvrages d'art ou ponctué de ponts et viaducs...?

Ce sont des problématiques de cette nature, que partagent des opérations d'inventaire sur d'autres réseaux techniques - les canaux, les routes, le transport de l'électricité...-, que la journée d'études a voulu mettre en débat, la récente régionalisation des services de l'Inventaire donnant à ce débat, comme on l'a dit, une nouvelle actualité. En effet, si le patrimoine ferroviaire, comme le patrimoine industriel ou le patrimoine de la villégiature balnéaire, semble requérir une vision nationale (pour ne pas dire internationale), les conseils régionaux, en tant qu'autorités organisatrices des transports ferroviaires, commencent à se montrer particulièrement sensibles aux aspects patrimoniaux de leurs réseaux, éventuellement porteurs d'une certaine identité régionale à valoriser. En même temps, et dans toutes les régions, les friches ferroviaires soulèvent des questions communes de reconversion et de développement urbain, durable, il va sans dire.

Après une séquence consacrée aux sources, la journée a donc cherché surtout à mettre en exergue, comme études de cas, quelques opérations récentes d'inventaire en Seine-Saint-Denis, en Franche-Comté et à Juvisy. Au cours de l'après-midi, autour de la Petite Ceinture à Paris, de la ligne (protégée au titre des monuments historiques) du Blanc-Argent et de la ligne touristique du Train Thur Doller Alsace de Cernay à Sentheim, la parole fut donnée à d'autres acteurs, essentiels, du patrimoine ferroviaire, le monde associatif. Bien évidemment, les actes de la journée publiés ici ne rendent qu'imparfaitement compte de la richesse des échanges qui suivirent ces communications ${ }^{7}$. Mais espérons que, imprimées, elles feront leur chemin, en enrichissant les réflexions à venir, en informant les actions d'inventaire à entreprendre et en confortant le réseau de compétences dont la journée se voulait l'amorce. Et rendons hommage, pour conclure, à l'efficacité, grande et habituelle, du secrétariat de l'AHICF dans l'organisation de la journée et dans la publication de ces actes.

7- Enregistrés, l'ensemble des débats de la journée peut être écouté sur le site de l'AHICF : http://www.ahicf.com/mp3-2008/actes2008.htm. 
